\title{
The Mediating Effect of Eveningness on the Indirect Relationships between Shorter Sleep Duration, Inattention, Depression with Smartphone Addiction Tendency
}

\author{
Yoonseok Kim', So-Jin Lee ${ }^{2,3}$, Chul-Soo Park ${ }^{2,3}$, Bong-Jo Kim², ${ }^{2,3}$ Cheol-Soon Lee ${ }^{3,4}$, Boseok Cha ${ }^{2,3}$, \\ Dongyun Lee, ${ }^{4}$ Jae-Won Choi ${ }^{2}$, Young-Ji Lee ${ }^{4}$, and Jiyeong Seo ${ }^{4}$ \\ 'Department of Psychiatry, Gyeongsang National University Graduate School of Medicine, Jinju, Korea \\ 2Department of Psychiatry, Gyeongsang National University Hospital, Jinju, Korea \\ ${ }^{3}$ Department of Psychiatry, School of Medicine, Gyeongsang National University, Jinju, Korea \\ ${ }^{4}$ Department of Psychiatry, Gyeongsang National University Changwon Hospital, Changwon, Korea
}

\begin{abstract}
Objective: Smartphones make our lives more convenient. To investigate the relationships between sleep-related habits, inattention, and smartphone addiction tendency (SAT), we determined how chronotype (morningness-eveningness) mediates these factors in Korean college students. Methods: Self-rated questionnaires including the Morningness-Eveningness Questionnaire, Smartphone Addiction Scale-Short Version, Adult Attention Deficit and Hyperactivity Disorder Scale, and Hospital Anxiety and Depression Scale were administered to 1,060 Korean college student smartphone users. This study investigated associations between sleep-related habits, social jetlag, inattention, anxiety, depression and SAT. A path analysis was conducted to evaluate the relationships, considering chronotype. Results: More social jetlag and smartphone use before bedtime had both direct and indirect relationships with SAT via eveningness in the path analysis model. Participants with shorter exposure to sunlight and more anxiety symptoms had a direct relationship with SAT. Individuals with a depressed mood, shorter sleep duration, and more inattentive features had no significant direct relationship with SAT but they had a significant indirect relationship with SAT via eveningness. Conclusion: The findings support the idea that chronotype plays a significant mediating role as a predictor of smartphone addiction. Managing modifiable variables and social jetlag as well as other clinical variables may help prevent and treat smartphone addiction in clinical practice.
\end{abstract}

Key Words: Cell phones; Behavior, addictive; Sleep; Circadian rhythm; Depression

Received: February 14, 2020 Revised: March 12, 2020 Accepted: March 13, 2020

Corresponding author: Jiyeong Seo, MD, Department of Psychiatry, Gyeongsang National University Changwon Hospital, 11 Samjeongja-ro, Seongsan-gu, Changwon 51472, Korea.

Tel: 82-55-214-3802, Fax: 82-55-214-3254, E-mail: guysheston@gmail.com

@ This is an Open Access article distributed under the terms of the Creative Commons Attribution Non-Commercial License (https://creativecommons.org/licenses/bync/4.0) which permits unrestricted non-commercial use, distribution, and reproduction in any medium, provided the original work is properly cited.

\section{INTRODUCTION}

Smartphones influence our personal lives, and smartphone users have increased explosively over the past decade. The proportion of adult smartphone users in 2015 was $88 \%$ in South Korea, $72 \%$ in United States, $77 \%$ in Australia, $68 \%$ in the UK, and 60\% in Italy [1]. Smartphones have features that are easily accessible and versatile. In addition to calling and texting, smartphones can also be used for document work, web browsing, health status checks, and taking photographs. According to a report by the Korea Internet and Security Agency, smartphone usage is highest among individuals in their 20s [2]. Hence, many studies have been performed on the use of smartphones by university and college students [3-10].

The use of a smartphone has many positive aspects; it allows information to be obtained easily via web browsing, as well as finding the best route to a destination using the GPS, and it can be used to control appliances. Furthermore, a previous study de- 
scribed some positive psychological functions of mobile phone usage [11]; it can help with long-distance relationships, dominate reality by controlling the present, and act as an antidepressant or anxiolytic to deal with isolation and loneliness. One study on smartphone usage patterns and frequency revealed that smartphones are used most for mobile instant messaging, web browsing, and social media [12]. Mobile instant messaging and social media can help with forming relationships and preventing isolation. This smartphone usage pattern is consistent with the positive psychological functions mentioned above.

Nevertheless, excessive smartphone use causes various problems. Overuse of a smartphone can increase the risk of car accidents [13], decrease productivity [14], make users less trustworthy [15] and produce musculoskeletal problems [16]. Reports about the negative aspects of smartphone overuse are growing annually. However, it remains controversial whether smartphone overuse is a behavioral addiction because smartphone overuse produces a variety of symptoms, and there is little neurobiological evidence to indicate a detrimental effect; furthermore, longitudinal studies are lacking. One study concluded that it was speculative to call smartphone overuse a behavioral addiction [17]. However, smartphone overuse can be explained by the five-elements of addiction [18], including behavior to eliminate uncomfortable or restless feelings, a preoccupation-like craving that only provides temporary satiation, loss of control, and negative consequences, including social disapproval and low self-esteem. The contents of smartphone addiction-related questionnaires have similarities with the criteria for gambling disorder, which is a newly included behavioral addictive disorder in the Diagnostic and Statistical Manual of Mental Disorders, Fifth Edition (DSM-5) and of internet gaming disorder, which can be found in the further study section of the DSM-5 [19]. In this context, more and more studies are regarding smartphone overuse as a behavioral addiction $[4,5,20,21]$.

Some studies have explored relationships between smartphone addiction and various factors to understand smartphone addiction more fully. Smartphone addiction has been associated with sleep disturbances [22], depression [3,23,24], anxiety [3,4,25], gender [26,27], alcohol abuse [6] and absolute smartphone usage time [20]. Among the many variables, the behaviorally modifiable variables, including sleep duration, sunlight exposure duration, and smartphone usage time before bedtime, as well as possibly modifiable variables, including social jetlag may be associated with smartphone addiction. Moreover, inattentive behavior can be related to a smartphone addiction because the smartphone has a variety of multitasking functions that offer short-term rewards. However, few studies have been conducted on inattention. In addition, few studies have investigated a direct relationship between smartphone addiction and chronotyp, although some studies have supported the idea that chronotype is associated with internet addiction, gaming addiction, and other clinical variables including anxiety, depression, and sleep pattern [8,28-33]. Thus, we hypothesized that chronotype has a mediating effect between smartphone addiction and clinical variables.
The aim of this study was to investigate relationships between sleep-related habits, inattention, and smartphone addiction tendency (SAT) as well as to evaluate how the chronotype mediates these variables in Korean college students. Moreover, we propose an effective method to prevent and treat smartphone addiction based on the results.

\section{METHODS}

\section{Participants}

This study was conducted as part of the Addiction Prevention Program for College Students by the Jinju Community Addiction Management Center. One study about morningness and resilience has been performed on this population [34]. The program was implemented from February to November 2014 using a self-report questionnaire. Participants in the program were students at the Science and Technology College, Jinju, Republic of Korea. A total of 1,093 adults [946 males and 147 females; age: $22.78 \pm 1.85$ years (mean $\pm \mathrm{SD}$ ); age range, 19-29 years] volunteered for the study. None of the participants had any serious medical or mental illness issues and no one was taking any medication. The Institutional Review Board of Gyeongsang National University Hospital approved this study (approval number: 2015-06-004-004).

\section{Instruments}

Most of the questionnaires were filled out by the participants [missing data ranged from $0 \%$ for the Adult Attention Deficit and Hyperactivity Disorder Scale (AADHDS) to 5.3\% for social jetlag]. The self-report questionnaires were Korean versions of survey rating scales, including the Korean version of the AADHDS [35], the Korean version of the Hospital Anxiety and Depression Scale (HADS) [36], the Korean version of the Morningness-Eveningness Questionnaire (MEQ) [37], the Smartphone Addiction ScaleShort Version (SAS-SV) [38] and a survey on social jetlag, which is defined as a discrepancy between social and endogenous time, sunlight exposure duration between 10:00 and 15:00 on weekdays and weekends, sleep duration, and smartphone usage time before bedtime.

\section{AADHDS}

The AADHDS is composed of 18 items that are equally divided between inattention and hyperactivity-impulsivity factors. Odd numbered questions measure inattention and even numbered questions measure hyperactivity-impulsivity. Each question scores 0-3 points. A higher score indicates a probable clinically significant condition of ADHD. This self-rating scale was developed by Murphy and Barkley [39] to evaluate adult ADHD based on the DSM-IV ADHD diagnostic criteria for children and adolescents. It has been used widely, as it can be rated easily based on the DSMIV ADHD diagnostic criteria. The Korean version of the AADHDS has good internal consistency and test-retest correlations [35]. In the present study, the Cronbach's alpha coefficient was 0.89. 


\section{HADS}

The HADS has been used widely to assess anxiety symptom severity and depressive disorders in a hospital setting and in the general population [40]. This scale is composed of 14 items divided into two subscales of anxiety (HAD-A) and depression (HAD-D) [41]. Each question scores $0-3$ points, and scores range from 0 to 21 for each subscale. A higher score represents a higher level of anxiety or depression. The Korean version of the HADS has been verified for validity and reliability [36]. In the present study, the Cronbach's alpha coefficient was 0.86 .

\section{SAS-SV}

The SAS-SV was developed to identify the level of risk for SAT, not for an addiction diagnosis. This scale is a shortened version of the original SAS. The original SAS is composed of 33 items [42] and the shortened version contains 10 items [38]. Each question scores 1-6 points. A higher score represents a higher risk for smartphone addiction. According to the scale developer, Kwon et al. [38], the cutoff scores of 31 for males and 33 for females indicate the high-risk smartphone addiction groups. The Korean version of the SAS-SV has been validated and is reliable [42]. In the present study, the Cronbach's alpha coefficient was 0.89 .

\section{MEQ}

The MEQ is composed of 19 items to classify individuals as morning-type and evening-type or the so-called chronotype [43]. This scale assesses the degree of alertness at certain times and the preference for sleep and awake time. According to Horne \& Ostberg, scores $\leq 41$ indicate evening types, and scores $\geq 59$ indicate morning types. The scale has been verified for validity and reliability [43]. The Korean version of the MEQ has also been verified for validity and reliability [37]. In the present study, the Cronbach's alpha coefficient was 0.69 .

\section{Other variables}

Additional survey items included social jetlag, which was defined as the discrepancy between social and endogenous time, calculated as the difference between weekday and weekend awakening times [44]. Exposure duration to sunlight was calculated, including the duration of any activities performed outside between 10:00 and 15:00 on weekdays and weekends. Activities included walking, exercising, or sitting on a bench. Sleep duration and smartphone usage time before bedtime were checked with subjective questions.

\section{Statistical analysis}

A single imputation was conducted using the expectation maximization algorithm to deal with missing data. The missing data ranged from $0 \%$ on the AADHDS to $5.1 \%$ for social jetlag. Little's MCAR test $\left(\chi^{2}=245.32, p=0.79\right)$ was not significant, indicating that the data were missing completely at random [45]. When data are missing completely at random and a very small portion of data are missing (i.e., $<5 \%$ ), the expectation maximization algorithm pro- vides unbiased parameter estimates [46,47]. Missing data were input using the missing value analysis in SPSS 21.0 (IBM Corp., Armonk, NY, USA). The independent t-test was performed to detect differences between the smartphone addiction risk groups and the clinical variables.

Pearson's correlation analysis was performed to evaluate the relationships between the SAS-SV and various clinical variables, including age, sex, inattention (component of AADHDS), HADA, HAD-D, MEQ, social jetlag, sunlight exposure duration, sleep duration, and smartphone usage before bedtime. The same analysis was performed to evaluate the relationships between the MEQ and various clinical variables, including age, sex, inattention (component of AADHDS), HAD-A, HAD-D, SAS-SV, social jetlag, sunlight exposure duration, sleep duration, and smartphone usage time before bedtime.

Significant variables from the correlation $(p<0.1)$ together with age, sex, depression, and anxiety were used as independent variables and the SAS-SV and MEQ scores were used as dependent variables in the multiple linear regression analysis. Finally, we performed a path analysis to estimate the relationship between clinical variables and smartphone addiction mediated by the chronotype. Multiple goodness-of-fit measures were used to assess model fit, including the chi-square, comparative-fit index (CFI), normedfit index (NFI), standardized root mean square residual (SRMR), root mean square error of approximation (RMSEA), adjusted goodness-of-fit statistic (AGFI), and the Tucker-Lewis Index (TLI). The correlation analysis, independent t-test, and multiple linear regression analysis were conducted using SPSS 21.0 for Windows. AMOS 18.0 (SPSS Inc., Chicago, IL, USA) was used for the path analysis. A two-tailed p-value $<0.05$ was considered statistically significant.

\section{RESULTS}

Of the 1,093 participants, 1,060 (96.98\%) were smartphone users. The 33 participants who did not use smartphones were excluded from the study. The participants' demographic and clinical characteristics are presented in Table 1. The average SAS-SV score was 21.03 ( $\mathrm{SD}=8.85$ ), and the average MEQ score was 45.09 $(\mathrm{SD}=7.68)$. Missing value range was $0 \%$ for the AADHDS to $5.1 \%$ for social jetlag. The missing data were input using the expectation maximization algorithm in SPSS 21.0.

The independent t-test was performed to identify differences between the smartphone normal-user group and smartphone addiction high-risk group. Kwon et al. [38] suggested different cutoff values by gender when predicting smartphone addiction. The smartphone addiction high-risk group was identified using the cut-off values of 31 for males and 33 for females. As shown in Table 2, significant differences were observed between the nor$\mathrm{mal}$ and the high-risk groups with regard to SAS-SV $(\mathrm{t}=-44.66$, $\mathrm{p}<0.001)$, MEQ ( $\mathrm{t}=3.35, \mathrm{p}=0.001)$, HAD-A ( $\mathrm{t}=-2.05, \mathrm{p}=0.041)$, inattention $(\mathrm{t}=-2.26, \mathrm{p}=0.025)$, sunlight exposure duration per day $(\mathrm{t}=2.19, \mathrm{p}=0.029)$, social jetlag $(\mathrm{t}=-3.56, \mathrm{p}<0.001)$, and smartphone 
usage time before bedtime $(\mathrm{t}=-5.83, \mathrm{p}<0.001)$. The HAD-D $(\mathrm{t}=$ -0.24, $\mathrm{p}=0.981)$ and sleep duration $(\mathrm{t}=0.068, \mathrm{p}=0.946)$ were not significantly different between the two groups.

Pearson's correlation analysis was performed to evaluate the association between clinical variables, each of the chronotypes (MEQ), and SAT (SAS-SV). The SAS-SV score was significantly correlated with age $(r=-0.108, p<0.001)$, sex $(r=0.103, p=0.001)$, inattention $(\mathrm{r}=0.11, \mathrm{p}<0.001)$, HAD-A $(\mathrm{r}=0.099, \mathrm{p}=0.001)$, MEQ $(\mathrm{r}=-0.123, \mathrm{p}<0.001)$, social jetlag $(\mathrm{r}=0.157, \mathrm{p}<0.001)$, sunlight $\mathrm{ex}-$ posure duration per day $(\mathrm{r}=-0.086, \mathrm{p}=0.005)$, and smartphone usage time before bedtime $(r=0.212, p<0.001)$. The HAD-D $(r=0.036$, $\mathrm{p}=0.246)$ and sleep duration $(\mathrm{r}=0.006, \mathrm{p}=0.834)$ were not significantly correlated with the SAS-SV score. The MEQ score was sig-

Table 1. Demographic and clinical characteristics $(n=1,060)$

\begin{tabular}{lr}
\multicolumn{1}{c}{ Variables } & Values \\
Sex & \\
Male & $918(86.6)$ \\
Female & $142(13.4)$ \\
Age, $y r$ & $22.78 \pm 1.85$ \\
HAD ( $\mathrm{n}=1,041)$ & $8.95 \pm 5.87$ \\
HAD-A $(\mathrm{n}=1,048)$ & $4.02 \pm 3.18$ \\
HAD-D ( $\mathrm{n}=1,043)$ & $4.93 \pm 3.33$ \\
MEQ (n=1,031) & $45.09 \pm 7.68$ \\
SAS-SV & $21.03 \pm 8.85$ \\
AADHDS & $7.25 \pm 6.71$ \\
Hyperactivity-impulsivity & $3.23 \pm 3.43$ \\
Inattention & $4.02 \pm 3.74$ \\
Exposure duration to sunlight per day, $\mathrm{hr}(\mathrm{n}=1,025)$ & $1.02 \pm 1.06$ \\
Social jetlag, hr (n=1,006) & $2.47 \pm 1.70$ \\
Sleep duration, hr ( $\mathrm{n}=1,043)$ & $6.36 \pm 1.67$ \\
Smartphone usage time before bedtime, $\mathrm{hr}(\mathrm{n}=1,040)$ & $0.66 \pm 0.83$ \\
\hline
\end{tabular}

Data are presented as mean \pm SD or number (percentage). HAD: Hospital Anxiety and Depression Scale, HAD-A: Hospital Anxiety and Depression Scale-Anxiety, HAD-D: Hospital Anxiety and Depression Scale-Depression, MEQ: Morningness-Eveningness Questionnaire, SAS-SV: Smartphone Addiction Scale-Short Version, AADHDS: Adult ADHD scale nificantly correlated with age $(\mathrm{r}=0.121, \mathrm{p}<0.001)$, sex $(\mathrm{r}=-0.091$, $\mathrm{p}=0.003)$, inattention $(\mathrm{r}=-0.264, \mathrm{p}<0.001)$, HAD-A $(\mathrm{r}=-0.181, \mathrm{p}<$ 0.001), HAD-D ( $\mathrm{r}=-0.241, \mathrm{p}<0.001)$, SAS-SV $(\mathrm{r}=-0.123, \mathrm{p}<0.001)$, social jetlag $(\mathrm{r}=-0.147, \mathrm{p}<0.001)$, sunlight exposure duration per day $(\mathrm{r}=0.078, \mathrm{p}<0.001)$, sleep duration $(\mathrm{r}=0.098, \mathrm{p}=0.001)$, and smartphone usage time before bedtime ( $\mathrm{r}=-0.092, \mathrm{p}=0.003)$.

A multiple linear regression analysis was conducted with clinical variables that were significantly associated with the SAS-SV and MEQ according to the Pearson's correlation analysis $(\mathrm{p}<0.1)$. One analysis was performed with SAS-SV as the dependent variable and with age, sex, HAD-A, HAD-D, MEQ, inattention, social jetlag, sunlight exposure duration per day, sleep duration, and smartphone usage time before bedtime as independent variables, and explained $8.6 \%$ of the variation in SAS-SV $(\mathrm{F}=10.995, \mathrm{p}<0.001$, adjusted $\left.R^{2}=0.086\right)$. HAD-A $(\beta=0.081, p=0.047)$, MEQ $(\beta=-0.065$, $\mathrm{p}=0.038)$, social jetlag $(\beta=0.110, \mathrm{p}<0.001)$, sunlight exposure duration per day $(\beta=-0.071, p=0.017)$, and smartphone usage time before bedtime $(\beta=0.196, p<0.001)$ were significant variables. In another analysis, we used MEQ as the dependent variable and age, sex, HAD-A, HAD-D, SAS-SV, inattention, social jetlag, sunlight exposure duration per day, sleep duration and smartphone usage time before bedtime as independent variables, and explained $11.8 \%$ of the variation in MEQ $\left(\mathrm{F}=15.166, \mathrm{p}<0.001\right.$, adjusted $\mathrm{R}^{2}=$ 0.118). The HAD-D $(\beta=-0.180, p<0.001)$, SAS-SV $(\beta=-0.063, p=$ $0.038)$, inattention $(\beta=-0.181, p<0.001)$, social jetlag $(\beta=-0.114$, $\mathrm{p}<0.001)$, sleep duration $(\beta=0.061, \mathrm{p}=0.039)$, and smartphone usage time before bedtime $(\beta=-0.068, p=0.021)$ had significant relationships.

A path analysis model was created to evaluate the relationships between clinical variables, the chronotype, and SAT. The MEQ was set to mediate the association between the clinical variables and SAS-SV in the model. Clinical variables that were significantly related with the SAS-SV and the MEQ according to the multiple linear regression analysis were used in the path analysis (Figure 1). Multiple goodness-of-fit measures were used to assess model fit, and all indices were a sufficient fit to the model; $\chi^{2}=9.353$ ( $\mathrm{df}=5$, $\mathrm{p}=0.096)$, $\mathrm{CFI}=0.996, \mathrm{NFI}=0.992, \mathrm{SRMR}=0.011, \mathrm{RMSEA}=0.029$,

Table 2. Comparison of variables related to smartphone addiction risk groups

\begin{tabular}{|c|c|c|c|c|}
\hline & $\begin{array}{l}\text { Smartphone normal user group } \\
\qquad(\mathrm{n}=930)\end{array}$ & $\begin{array}{l}\text { Smartphone addiction high risk group } \\
\qquad(\mathrm{n}=130)\end{array}$ & $\mathrm{t}$ & p-value \\
\hline SAS-SV & $18.33(6.38)$ & $36.21(4.29)$ & -44.66 & $<0.001$ \\
\hline MEQ & $45.4(7.55)$ & $43.25(7.58)$ & 3.35 & 0.001 \\
\hline HAD-A & $3.93(3.19)$ & $4.48(3.02)$ & -2.05 & 0.041 \\
\hline HAD-D & $4.92(3.33)$ & $4.93(3.27)$ & -0.24 & 0.981 \\
\hline PSQI & $4.92(2.69)$ & $5.11(2.73)$ & 1.59 & 0.413 \\
\hline Inattention (component of AADHDS) & $3.90(3.64)$ & $4.70(4.21)$ & -2.26 & 0.025 \\
\hline Exposure duration to sunlight per day & $1.05(1.05)$ & $0.85(1.01)$ & 2.19 & 0.029 \\
\hline Social jetlag & $2.40(1.67)$ & $2.90(1.52)$ & -3.56 & $<0.001$ \\
\hline Sleep duration & $6.35(1.65)$ & $6.34(1.74)$ & 0.068 & 0.946 \\
\hline Smartphone usage time before bedtime & $0.59(0.79)$ & $1.02(0.88)$ & -5.83 & $<0.001$ \\
\hline
\end{tabular}

Data are presented as mean (SD). HAD: Hospital Anxiety and Depression Scale, HAD-A: Hospital Anxiety and Depression Scale-Anxiety, HADD: Hospital Anxiety and Depression Scale-Depression, MEQ: Morningness-Eveningness Questionnaire, AADHDS: Adult ADHD scale, SAS-SV: Smartphone Addiction Scale-Short Version, SD: standard deviation 
AGFI $=0.982$, TLI $=0.973$. As shown in Table 3 , all path coefficients were significant. Table 4 depicts the causal effect that included direct, indirect, and total effects of the clinical variables on SAT. The path from smartphone usage time before bedtime and social jetlag to SAT had both direct and indirect effects. Spending more time using a smartphone before bedtime and experiencing a larger social jetlag were associated with higher SAT. Additionally, the total effects of both variables increased slightly when MEQ mediated the associations. The indirect path from the HAD-D, sleep duration, and inattention to SAT mediated by the MEQ were significant. Experiencing more depressive symptoms, shorter sleep duration, and being a more inattentive type were related to the eveningness and led to a high risk of smartphone addiction. The direct path from $\mathrm{HAD}$-A and sunlight exposure duration to SAT was significant. Being more anxious and spending less time in sunlight suggested a higher SAT.

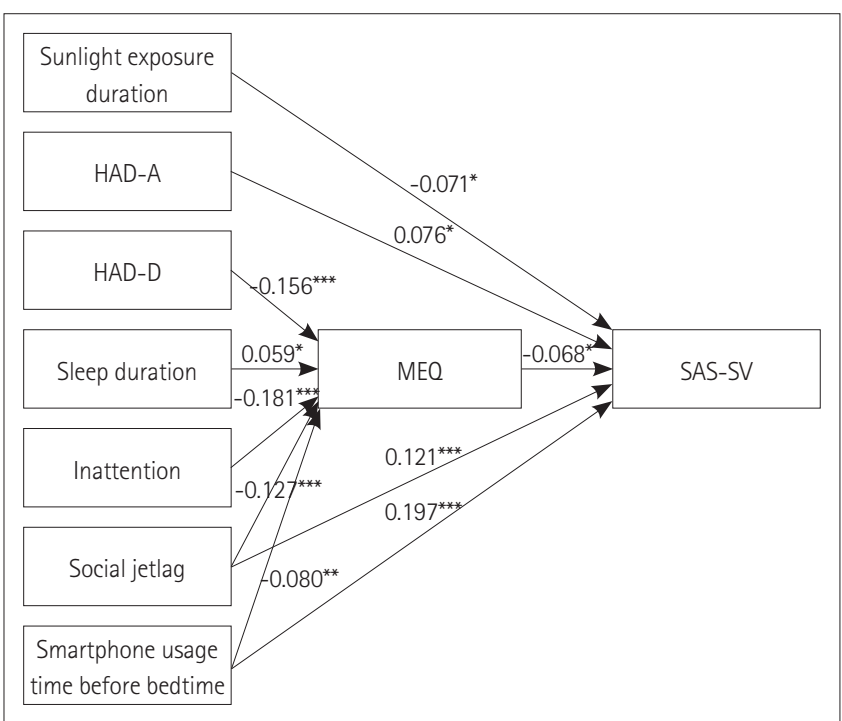

Figure 1. Path model of the relationship between the MEQ, SAS-SV, and other variables. MEQ: Morningness-Eveningness Questionnaire, SAS-SV: Smartphone Addiction Scale-Short Version, HAD-A: Hospital Anxiety and Depression Scale-Anxiety, HAD-D: Hospital Anxiety and Depression Scale-Depression. ${ }^{*} p<0.05,{ }^{* *} p=0.006,{ }^{* * *} p<0.01$.

\section{DISCUSSION}

The present study demonstrated that more social jetlag and longer smartphone usage before bedtime had both direct and indirect relationships with high SAT via eveningness. Moreover, shorter exposure to sunlight and more anxiety symptoms had direct relationships with high SAT. In contrast, more depressive symptoms, shorter sleep duration, and more symptoms of inattention had an indirect relationship with high SAT through the eveningness.

The present study identified that the chronotype had a direct relationship with SAT. A negative relationship was observed between morningness and SAT, which is consistent with a previous study [48]. Chronotype is a phenotype that results from a person's genetics interacting with the environment [49]. In particular, it is known that eveningness is associated with addiction proneness including internet addiction $[8,28]$ and computer game addiction [31]. Internet use and games are very common smartphone activities. Moreover, several studies have revealed that eveningness individuals are related to substance use problems that can be the beginning of addiction. Eveningness individuals have a tendency

Table 4. Standardized direct, indirect, and total effects of clinical variables on smartphone addiction tendency

\begin{tabular}{lccc}
\multirow{2}{*}{ Variables } & \multicolumn{3}{c}{ Effect } \\
\cline { 2 - 4 } & Direct & Indirect & Total \\
Smartphone usage time before bedtime & 0.197 & 0.005 & 0.202 \\
Social jetlag & 0.121 & 0.009 & 0.130 \\
HAD-A & 0.076 & - & 0.076 \\
Exposure duration to sunlight per day & -0.071 & - & -0.071 \\
MEQ & -0.068 & - & -0.068 \\
Inattention & - & 0.012 & 0.012 \\
HAD-D & - & 0.011 & 0.011 \\
Sleep duration & - & -0.004 & -0.004 \\
\hline
\end{tabular}

HAD-A: Hospital Anxiety and Depression Scale-Anxiety, HAD-D: Hospital Anxiety and Depression Scale-Depression, MEQ: Morningness-Eveningness Questionnaire, SAS-SV: Smartphone Addiction Scale-Short Version

Table 3. Standardized coefficients, standard error, critical ratio, and p-values for each path

\begin{tabular}{|c|c|c|c|c|c|}
\hline & & Standardized coefficients & Standard error & Critical ratio & p-value \\
\hline MEQ & $\leftarrow$ Sleep duration & 0.059 & 0.133 & 2.016 & 0.044 \\
\hline MEQ & $\leftarrow$ HAD-D & -0.156 & 0.074 & -4.861 & $<0.010$ \\
\hline MEQ & $\leftarrow$ Inattention & -0.181 & 0.065 & -5.633 & $<0.010$ \\
\hline MEQ & $\leftarrow$ Social jetlag & -0.127 & 0.133 & -4.391 & $<0.010$ \\
\hline MEQ & $\leftarrow$ Smartphone usage time before bedtime & -0.080 & 0.268 & -2.756 & 0.006 \\
\hline SAS-SV & $\leftarrow$ Exposure duration to sunlight per day & -0.071 & 0.250 & -2.393 & 0.017 \\
\hline SAS-SV & $\leftarrow$ HAD-A & 0.076 & 0.084 & 2.517 & 0.012 \\
\hline SAS-SV & $\leftarrow$ Smartphone usage time before bedtime & 0.197 & 0.319 & 6.640 & $<0.010$ \\
\hline SAS-SV & $\leftarrow$ MEQ & -0.068 & 0.036 & -2.240 & 0.025 \\
\hline SAS-SV & $\leftarrow \quad$ Social jetlag & 0.121 & 0.160 & 4.059 & $<0.010$ \\
\hline
\end{tabular}

Fit of path model: $\chi^{2}=9.353, \mathrm{df}=5$, p-value $=0.096, \mathrm{CFI}=0.996, \mathrm{NFI}=0.992, \mathrm{SRMR}=0.011, \mathrm{RMSEA}=0.029, \mathrm{AGFI}=0.982$, TLI=0.973. CFI: comparative-fit index, NFI: normed-fit index, SRMR: standardized root mean square residual, RMSEA: root mean square error of approximation, AGFI: adjusted goodness-of-fit statistic index, TLO: Tucker-Lewis Index, HAD-A: Hospital Anxiety and Depression Scale-Anxiety, HAD-D: Hospital Anxiety and Depression Scale-Depression, MEQ: Morningness-Eveningness Questionnaire, SAS-SV: Smartphone Addiction Scale-Short Version 
to consume more psychoactive drugs, smoke, and drink more alcohol than morningness individuals [50,51]. Many studies have provided evidence that abnormalities in circadian rhythms may be associated to susceptibility to addiction $[52,53]$. For example, recent cross-sectional studies of inpatient clinics have shown that addiction disorders are more likely to be evening type than other patients [54]. In the research on neural mechanisms of these relationships, eveningness is associated with reduced in medial prefrontal cortex reactivity that reflects reward-related brain function in adolescents. These findings are consistent with a main result of our study [55].

In the present study, social jetlag and smartphone usage time before bedtime had both direct and indirect relationships with SAT. Social jetlag represents the discrepancy between biological and social time [56]. In this study, social jetlag was calculated as the difference between weekday and weekend rise times [44]. No previous studies have investigated the relationship between social jetlag and SAT. The higher the social jetlag, the greater the risk for SAT. Furthermore, social time disrupts circadian rhythms [57]. Additionally, many studies have suggested that disrupting circadian rhythms is related to addiction, such as to alcohol and cocaine $[58,59]$. Similarly, some studies have reported that disrupting circadian rhythms enhances the addiction risk by altering reward-related brain function $[60,61]$. Although the effect was small, social jetlag had a significant indirect effect, with SAT being mediated by chronotype in our study. Smartphone usage time before bedtime also had direct and indirect relationships with SAT. Smartphone usage time is one of the most important indicators and is included in almost all smartphone addiction scales $[5,38,42,62]$. Our results are in line with a previous study reporting that a smartphone addicted group used their smartphones more at night than a non-addicted group [12]. In addition, smartphone usage time before bedtime is related with eveningness $[9,63]$ and exposure to the blue light of a smartphone has a melatonin suppressing effect $[64,65]$ that might make individuals prefer evening activities [66]. In a previous study of a relationship between sleep patterns and use of electronic media in bed have shown that using the internet before bedtime significantly associated with bedtime delay [67]. They explained that computer screen light could affect circadian rhythms, resulting in syndrome sleep phase delay. This suggests that the use of smartphone before bedtime can similarly affect sleep phase delay by exposure to bright and blue light of smartphone. However, further research will be needed to understand related mechanisms. Also, our results show that smartphone usage time before bedtime was indirectly associated with SAT via chronotype. Thus, a reduction in social jetlag and nighttime smartphone use are important factors to consider when trying to prevent smartphone addiction.

The present study found that anxiety symptoms and sunlight exposure duration had direct relationships with SAT. The relationship between anxiety symptoms and SAT is highly consistent with previous studies. Individuals who feel more anxious have a tendency to become smartphone addicted $[3,4,7,10,25,68]$. How- ever, there are various kinds of anxiety that have been measured by different studies, such as general anxiety, interpersonal anxiety, and social anxiety $[69,70]$. Smartphone users engage in social media, mobile instant messaging, web browsing, and playing games. Some users feel isolated and withdrawn from the group when they do not get a reply from an instant message or a social media post, which can cause another kind of anxiety. We only measured general anxiety using the HAD-A. Future studies should evaluate various aspects of anxiety symptoms. Sunlight exposure duration was negatively correlated with SAT. Serotonin $(5-\mathrm{HT})$ has various roles in relation to motivation, eating, emotions, moods, and cognition. Thus, 5-HT may play an important role in addiction [71]. Previous studies have revealed that addictive behavior with alcohol [72] and pathological gambling [73] are associated with lower 5-HT levels. In addition, several studies have reported that light exposure increases blood 5-HT levels in a specific brain region in humans and rats [74-77]. Hence, we speculate that 5-HT mediated the negative correlation between sunlight exposure and SAT. Further experimental studies will be needed to elucidate these relationships.

The present findings indicate that depressive symptoms, sleep duration, and inattention have indirect relationships with SAT that were mediated by chronotype. Several studies have shown that depression and smartphone addiction are significantly related $[3,6,70,78,79]$, whereas other studies have not $[4,24]$. The latter studies are in line with the results of our study. The use of smartphones can be used to alleviate negative emotions and depression [80]. Thus, smartphone overuse may have masked the direct relationship between smartphone addiction and depression in this study. At the same time, depression had an indirect effect on SAT mediated by chronotype. Previous studies have reported that depressive symptoms are more frequent in evening-types than in other circadian types [29]. Another study also revealed fewer morning types among subjects with depression than in a control group [30]. The indirect relationship between depression and SAT can be explained in that way. Several studies were in accordance with our sleep duration results. One study reported that SAS scores are not correlated with sleep duration but significantly correlated with sleep quality in Turkish university students [3]. A study of smartphone addiction in Korean university and graduate students also showed no significant relationship between sleep duration and smartphone addiction [22]. However, the regression model used in a study on the American general population reported that longer than average smartphone screen time is significantly associated with shorter sleep duration after adjusting for clinical variables [81]. Further study will be needed depending on the target population to reveal the direct relationship between sleep duration and smartphone addiction. In contrast, the present findings indicate that sleep duration had an indirect effect on SAT through chronotype. Shorter sleep duration was associated with the eveningness chronotype in previous studies $[82,83]$. Our path analysis (Figure 1) showed that shorter sleep duration was associated with eveningness and that it affects SAT. In the present study, 
inattention had no direct relationship with SAT, but it had an indirect relationship through chronotype. Although there is a report that patients with ADHD have a high frequency of SAT [23], few studies have investigated the relationship between inattention and SAT in the general population. Inattention was associated with time spent on a mobile phone in Chinese adolescents [84], but there are few studies on the relationship between inattention and the SAT in university students. One study reported that the inattentive domain of ADHD is associated with eveningness [85], which supports the idea that inattention is indirectly related to SAT through chronotype.

These findings suggest methods that can be applied to clinical practice. For example, an individual's chronotype may help clinicians manage smartphone-addicted patients. Additionally, clinicians should consider and mention behaviorally modifiable variables, including suggestions regarding extending sunlight exposure, avoiding short sleep times, reducing smartphone use before bedtime and understanding the concept of social jetlag, to smartphoneaddicted patients.

Several limitations of this study should be discussed. First, this was a cross-sectional study, so causal relationships could not be evaluated. Nevertheless, we tried to investigate causality between SAT and the clinical variables using a statistical method that included multiple linear regression and path analyses. Further research should be designed as a prospective cohort study to examine the relationships between other potential clinical variables and SAT. Second, all participants were college students and may not represent the general population. College students are well-educated young adults. Hence, they may access smartphones more easily than older people, which could lead to a greater addiction tendency. This said, studying younger people could be more helpful to our understanding of the pathophysiology of smartphone addiction because young adults in their 20 s are the most frequent users of smartphones, as compared to other age groups. Third, the region where the questionnaire was conducted was a small city in Korea. This region does not represent the entire nation, including the prevalence of addiction. Indeed, the problem of addiction in this region was not particularly high. According to Statistics Korea data from 2017, alcoholism was within 2 SD and heavy smoking was within $1 \mathrm{SD}$, as compared to the average in this area. Last, the research participants were biased toward males due to the characteristics of a technology college. Several studies have been performed on gender differences in smartphone addiction [3,6,48]. Hence, the ratio of males and females should be considered in further studies.

Despite these limitations, a strength of this study was that the relationship between the clinical variables and smartphone addiction was examined considering chronotype. The present study will provide clinicians with a better understanding of smartphonerelated problems and suggest methods that can be applied to clinical practice. Moreover, well-structured programs can be created that reduce smartphone addiction by controlling modifiable variables. Future research is needed to test whether smartphone ad- diction can be alleviated when social jetlag is reduced, sunlight exposure duration is increased, sleep duration is controlled, subjects stop using their smartphone before bedtime, and chronotype is considered individually.

\section{Acknowledgments}

This study was supported by a grant from the Korean Mental Health Technology R\&D Project, Ministry of Health \& Welfare, Republic of Korea (HM15C1108).

\section{Conflicts of Interest}

The authors have no potential conflicts of interest to disclose.

\section{Author Contributions}

Conceptualization: Yoonseok Kim. Data curation: Yoonseok Kim, So-Jin Lee, Chul-Soo Park. Formal analysis: Yoonseok Kim. Funding acquisition: So-Jin Lee. Investigation: Yoonseok Kim, So-Jin Lee. Methodology: Yoonseok Kim, Dongyun Lee. Project administration: Jiyeong Seo. Resources: Jiyeong Seo, Chul-Soo Park. Software: So-Jin Lee, Young-Ji Lee. Supervision: Bong-Jo Kim, Cheol-Soon Lee, Boseok Cha, Jiyeong Seo. Validation: Dongyun Lee, Young-Ji Lee. Visualization: Yoonseok Kim, JaeWon Choi. Writing-original draft: Yoonseok Kim. Writingreview \& editing: So-Jin Lee, Jiyeong Seo.

\section{ORCID iDs}

Jiyeong Seo (1)

https://orcid.org/0000-0002-7329-8296

Yoonseok Kim (1)

https://orcid.org/0000-0001-9245-6977

\section{REFERENCES}

1. Poushter J. Smartphone ownership and internet usage continues to climb in emerging economies. Washington, DC: Pew Research Center; 2016.

2. Korea Internet and Security Agency. 2016 survey on the internet usage. Available at: https://www.kisa.or.kr/eng/usefulreport/surveyReport_View. jsp $?$ cPage $=1 \&$ p_No $=262 \& b \_N o=262 \& d \_N o=80 \& S T=\& S V=$. Accessed March 14, 2020.

3. Demirci K, Akgönül M, Akpinar A. Relationship of smartphone use severity with sleep quality, depression, and anxiety in university students. J Behav Addict 2015;4:85-92.

4. Mok JY, Choi SW, Kim DJ, Choi JS, Lee J, Ahn H, et al. Latent class analysis on internet and smartphone addiction in college students. Neuropsychiatr Dis Treat 2014;10:817-828.

5. Tossell C, Kortum P, Shepard C, Rahmati A, Zhong L. Exploring smartphone addiction: insights from long-term telemetric behavioral measures. iJIM 2015;9:37-43.

6. Choi SW, Kim DJ, Choi JS, Ahn H, Choi EJ, Song WY, et al. Comparison of risk and protective factors associated with smartphone addiction and Internet addiction. J Behav Addict 2015;4:308-314.

7. Lepp A, Barkley JE, Karpinski AC. The relationship between cell phone use, academic performance, anxiety, and Satisfaction with Life in college students. Comput Hum Behav 2014;31:343-350.

8. Randler C, Horzum MB, Vollmer C. Internet Addiction and Its Relationship to Chronotype and Personality in a Turkish University Student Sample. Soc Sci Comput Rev 2014;32:484-495.

9. Lee U, Lee J, Ko M, Lee C, Kim Y, Yang S, et al. Hooked on smartphones: an exploratory study on smartphone overuse among college students. Proceedings of the SIGCHI Conference on Human Factors in Computing Sys- 
tems. New York, NY: Association for Computing Machinery; 2014. p. 23272336.

10. Jenaro C, Flores N, Gómez-Vela M, González-Gil F, Caballo C. Problematic internet and cell-phone use: Psychological, behavioral, and health correlates. Addict Res Theory 2007;15:309-320.

11. Peraman R, Parasuraman S. Mobile phone mania: arising global threat in public health. J Nat Sci Biol Med 2016;7:198-200.

12. Ahn H, Wijaya ME, Esmero BC. A systemic smartphone usage pattern analysis: focusing on smartphone addiction issue. Int J Multimed Ubiquitous Eng 2014;9:9-14.

13. Basacik D, Reed N, Robbins R. Smartphone use while driving: a simulator study. London, UK: Transport Research Laboratory; 2011. Published Project Report: PPR 592.

14. Kushlev K, Proulx J, Dunn EW. "Silence Your Phones”: Smartphone Notifications Increase Inattention and Hyperactivity Symptoms. Proceedings of the 2016 CHI Conference on Human Factors in Computing Systems (CHI '16). New York, NY: Association for Computing Machinery; 2016. p. 10111020.

15. Krishnan A, Kurtzberg TR, Naquin CE. The curse of the smartphone: electronic multitasking in negotiations. Negot J 2014;30:191-208.

16. Kim H, Kim JS. The relationship between smartphone use and subjective musculoskeletal symptoms and university students. J Phys Ther Sci 2015; 27:575-579.

17. Billieux J, Maurage P, Lopez-Fernandez O, Kuss DJ, Griffiths md et al. Can disordered mobile phone use be considered a behavioral addiction? An update on current evidence and a comprehensive model for future research. Curr Addict Rep 2015;2:156-162.

18. Sussman S, Sussman AN. Considering the definition of addiction. Int J Environ Res Public Health 2011;8:4025-4038.

19. Americal Psychiatric Association. Diagnostic and Statistical Manual of Mental Disorders (DSM- $\left.5^{\circledR}\right)$. 5th ed. Arlington, VA: American Psychiatric Association; 2013.

20. Haug S, Castro RP, Kwon M, Filler A, Kowatsch T, Schaub MP. Smartphone use and smartphone addiction among young people in Switzerland. J Behav Addict 2015;4:299-307.

21. Samaha M, Hawi NS. Relationships among smartphone addiction, stress, academic performance, and satisfaction with life. Comput Hum Behav 2016;57:321-325.

22. Heo JY, Kim SH, Han MA, Ahn YJ. Correlation between smartphone addiction and quality of sleep among university school students, graduate students. J Korean Inst Electr Commun Sci 2015;10:737-748.

23. Lee HC, Hong MH, Oh CK, Shim SH, Jun YJ, Lee SB, et al. Smart-phone addiction, depression/anxiety, and self-esteem with attention-deficit hyperactivity disorder in Korean children. J Korean Acad Child Adolesc Psychiatry 2015;30;26:159-164.

24. Kim SM, Huh HJ, Cho H, Kwon M, Choi JH, Ahn HJ, et al. The effect of depression, impulsivity, and resilience on smartphone addiction in university students. J Korean Neuropsychiatr Assoc 2014;53:214-220.

25. Hong FY, Chiu SI, Huang DH. A model of the relationship between psychological characteristics, mobile phone addiction and use of mobile phones by Taiwanese university female students. Comput Hum Behav 2012;28: 2152-2159.

26. Al-Barashdi HS, Bouazza A, Jabur NH. Smartphone addiction among university undergraduates: a literature review. J Sci Res Rep 2015;4:210-225.

27. Kim Y, Jeong JE, Cho H, Jung DJ, Kwak M, Rho MJ, et al. Personality factors predicting smartphone addiction predisposition: behavioral inhibition and activation systems, impulsivity, and self-control. PLoS One 2016;11: e0159788.

28. Kang DW, Soh M, Lee TK. Relationship between internet addiction and circadian rhythm in adults. Sleep Med Psychophysiol 2015;22:57-63.

29. Merikanto I, Lahti T, Kronholm E, Peltonen M, Laatikainen T, Vartiainen E, et al. Evening types are prone to depression. Chronobiol Int 2013;30:719725 .

30. Bielen J, Melada A, Markelić I. Depression and circadian typology. Psychiatr Danub 2015;27:190-192.

31. Vollmer C, Randler C, Horzum MB, Ayas T. Computer game addiction in adolescents and its relationship to chronotype and personality. SAGE Open 2014 Jan 10 [Epub]. Available from: https://doi.org/10.1177/2158244013518054.

32. Arrona-Palacios A, García A, Valdez P. Sleep-wake habits and circadian preference in Mexican secondary school. Sleep Med 2015;16:1259-1264.

33. Díaz-Morales JF, Sánchez-López MP. Morningness-eveningness and anxiety among adults: a matter of sex/gender? Pers Individ Dif 2008;44:13911401.

34. Lee SJ, Park CS, Kim BJ, Lee CS, Cha B, Lee YJ, et al. Association between morningness and resilience in Korean college students. Chronobiol Int 2016;33:1391-1399.

35. Kim EJ. The validation of Korean adult ADHD scale (K-AADHDS). Korean J Clin Psychol 2003;22:897-911.

36. Oh SM, Min KJ, Park DB. A study on the standardization of the Hospital Anxiety and Depression Scale for Koreans: a comparison of normal, depressed and anxious groups. J Korean Neuropsychiatr Assoc 1999;38:289296.

37. Yu NJ, Shin SC, Wang SK. A study on the standardization of the Korean version of JA Horne and O. Ostbergs' Morningness-Eveningness Questionnaire and on the sleep pattern. J Korean Neuropsychiatr Assoc 1995;34 642-656.

38. Kwon M, Kim DJ, Cho H, Yang S. The Smartphone Addiction Scale: development and validation of a short version for adolescents. PLoS One 2013; 8:e83558.

39. Murphy K, Barkley RA. Preliminary normative data on DSM-IV criteria for adults. ADHD report 1995;3:6-7.

40. Bjelland I, Dahl AA, Haug TT, Neckelmann D. The validity of the Hospital Anxiety and Depression Scale: an updated literature review. J Psychosom Res 2002;52:69-77.

41. Zigmond AS, Snaith RP. The Hospital Anxiety and Depression Scale. Acta Psychiatr Scand 1983;67:361-370.

42. Kwon M, Lee JY, Won WY, Park JW, Min JA, Hahn C, et al. Development and validation of a smartphone addiction scale (SAS). PLoS One 2013;8: e56936.

43. Horne JA, Ostberg O. A self-assessment questionnaire to determine morningness-eveningness in human circadian rhythms. Int J Chronobiol 1976;4: 97-110.

44. Randler C. Proactive people are morning people. J Appl Soc Psychol 2009; 39:2787-2797.

45. Little RJA. A test of missing completely at random for multivariate data with missing values. J Am Stat Assoc 1988;83:1198-1202.

46. Enders CK. A primer on maximum likelihood algorithms available for use with missing data. Struct Equ Modeling 2001;8:128-141.

47. Scheffer J. Dealing with missing data. Res Lett Inf Math Sci 2002;3:153-160.

48. Randler C, Wolfgang L, Matt K, Demirhan E, Horzum MB, Beşoluk Ş. Smartphone addiction proneness in relation to sleep and morningnesseveningness in German adolescents. J Behav Addict 2016;5:465-473.

49. Roenneberg T, Wirz-Justice A, Merrow M. Life between clocks: daily temporal patterns of human chronotypes. J Biol Rhythms 2003;18:80-90.

50. Gau SS, Shang CY, Merikangas KR, Chiu YN, Soong WT, Cheng AT. Association between morningness-eveningness and behavioral/emotional problems among adolescents. J Biol Rhythms 2007;22:268-274.

51. Adan A. Chronotype and personality factors in the daily consumption of alcohol and psychostimulants. Addiction 1994;89:455-462.

52. Gulick D, Gamsby JJ. Racing the clock: the role of circadian rhythmicity in addiction across the lifespan. Pharmacol Ther 2018;188:124-139.

53. Meyrel M, Rolland B, Geoffroy PA. Alterations in circadian rhythms following alcohol use: a systematic review. Prog Neuropsychopharmacol Biol Psychiatry 20;99:109831.

54. Lemoine P, Zawieja P, Ohayon MM. Associations between morningness/ eveningness and psychopathology: an epidemiological survey in three inpatient psychiatric clinics. J Psychiatr Res 2013;47:1095-1098.

55. Hasler BP, Sitnick SL, Shaw DS, Forbes EE. An altered neural response to reward may contribute to alcohol problems among late adolescents with an evening chronotype. Psychiatry Res 2013;214:357-364.

56. Wittmann M, Dinich J, Merrow M, Roenneberg T. Social jetlag: misalignment of biological and social time. Chronobiol Int 2006;23:497-509.

57. Foster RG, Peirson SN, Wulff K, Winnebeck E, Vetter C, Roenneberg T. Sleep and circadian rhythm disruption in social jetlag and mental illness. Prog Mol Biol Transl Sci 2013;119:325-346.

58. Bildt $\mathrm{C}$, Michélsen H. Gender differences in the effects from working conditions on mental health: a 4-year follow-up. Int Arch Occup Environ Health 2002;75:252-258. 
59. Abarca C, Albrecht U, Spanagel R. Cocaine sensitization and reward are under the influence of circadian genes and rhythm. Proc Natl Acad Sci U S A 2002;99:9026-9030.

60. Logan RW, Williams WP 3rd, McClung CA. Circadian rhythms and addiction: mechanistic insights and future directions. Behav Neurosci 2014;128: $387-412$.

61. Hasler BP, Clark DB. Circadian misalignment, reward-related brain function, and adolescent alcohol involvement. Alcohol Clin Exp Res 2013;37: 558-565.

62. Kim D, Lee Y, Lee J, Nam JK, Chung Y. Development of Korean Smartphone Addiction Proneness Scale for youth. PLoS One 2014;9:e97920.

63. Fossum IN, Nordnes LT, Storemark SS, Bjorvatn B, Pallesen S. The association between use of electronic media in bed before going to sleep and insomnia symptoms, daytime sleepiness, morningness, and chronotype. Behav Sleep Med 2014;12:343-357.

64. West KE, Jablonski MR, Warfield B, Cecil KS, James M, Ayers MA, et al. Blue light from light-emitting diodes elicits a dose-dependent suppression of melatonin in humans. J Appl Physiol (1985) 2011;110:619-626.

65. Figueiro M, Overington D. Self-luminous devices and melatonin suppression in adolescents. Lighting Res Technol 2016;48:966-975.

66. Harada T, Morikuni M, Yoshii S, Yamashita Y, Takeuchi H. Usage of mobile phone in the evening or at night makes Japanese students evening-typed and night sleep uncomfortable. Sleep Hypn 2002;4:150-153.

67. Oka Y, Suzuki S, Inoue Y. Bedtime activities, sleep environment, and sleep/ wake patterns of Japanese elementary school children. Behav Sleep Med 2008;6:220-233.

68. Elhai JD, Dvorak RD, Levine JC, Hall BJ. Problematic smartphone use: a conceptual overview and systematic review of relations with anxiety and depression psychopathology. J Affect Disord 2017;207:251-259.

69. Lee YK, Chang CT, Lin Y, Cheng ZH. The dark side of smartphone usage: Psychological traits, compulsive behavior and technostress. Comput Hum Behav 2014;31:373-383.

70. Ha JH, Chin B, Park DH, Ryu SH, Yu J. Characteristics of excessive cellular phone use in Korean adolescents. Cyberpsychol Behav 2008;11:783-784.

71. Müller CP, Homberg JR. The role of serotonin in drug use and addiction. Behav Brain Res 2015;277:146-192.

72. George AK, Paul J, Kaimal SB, Paulose CS. Decreased cerebral cortex and liver 5-HT2A receptor gene expression and enhanced ALDH activity in ethanol-treated rats and hepatocyte cultures. Neurol Res 2010;32:510-518.

73. Nordin C, Eklundh T. Altered CSF 5-HIAA disposition in pathologic male gamblers. CNS Spectr 1999;4:25-33.

74. Carlsson A, Svennerholm L, Winblad B. Seasonal and circardian monoamine variations in human brains examined post mortem. Acta Psychiatr Scand 1980;61(S280):75-85.

75. Rao ML, Müller-Oerlinghausen B, Mackert A, Strebel B, Stieglitz RD, Volz HP. Blood serotonin, serum melatonin and light therapy in healthy subjects and in patients with nonseasonal depression. Acta Psychiatr Scand 1992; 86:127-132.

76. Ferraro JS, Steger RW. Diurnal variations in brain serotonin are driven by the photic cycle and are not circadian in nature. Brain Res 1990;512:121124.

77. Cagampang FR, Yamazaki S, Otori Y, Inouye SI. Serotonin in the raphe nuclei: regulation by light and an endogenous pacemaker. Neuroreport 1993; 5:49-52.

78. Smetaniuk P. A preliminary investigation into the prevalence and prediction of problematic cell phone use. J Behav Addict 2014;3:41-53.

79. Thomée S, Härenstam A, Hagberg M. Mobile phone use and stress, sleep disturbances, and symptoms of depression among young adults--a prospective cohort study. BMC Public Health 2011;11:66.

80. Kim JH, Seo M, David P. Alleviating depression only to become problematic mobile phone users: can face-to-face communication be the antidote? Comput Hum Behav 2015;51:440-447.

81. Christensen MA, Bettencourt L, Kaye L, Moturu ST, Nguyen KT, Olgin JE, et al. Direct measurements of smartphone screen-time: relationships with demographics and sleep. PLoS One 2016;11:e0165331.

82. Allebrandt KV, Teder-Laving M, Kantermann T, Peters A, Campbell H, Rudan I, et al. Chronotype and sleep duration: the influence of season of assessment. Chronobiol Int 2014;31:731-740.

83. Merikanto I, Kronholm E, Peltonen M, Laatikainen T, Lahti T, Partonen T. Relation of chronotype to sleep complaints in the general Finnish population. Chronobiol Int 2012;29:311-317.

84. Zheng F, Gao P, He M, Li M, Wang C, Zeng Q, et al. Association between mobile phone use and inattention in 7102 Chinese adolescents: a population-based cross-sectional study. BMC Public Health 2014;14:1022.

85. Caci H, Bouchez J, Baylé FJ. Inattentive symptoms of ADHD are related to evening orientation. J Atten Disord 2009;13:36-41. 